\title{
Video Streaming Compression for Wireless Multimedia Sensor Networks
}

\author{
Nethra B.M ${ }^{1}$, Mr. Sudhakar K.N ${ }^{2}$ \\ ${ }^{1}$ CMRIT ,Bangalore.India \\ ${ }^{2}$ Associate Professor, Dept, Of CSE, CMRIT.Bangalore.
}

\begin{abstract}
This Article Presents The Design Of Continuous Video Streaming Compression Of A Networked System For Joint Compression, Error Detection And For Error Correction. Video Compression Applications Are Becoming More Popular Over Wireless Multimedia Sensor Networks. To Save Energy In Bit Transmissions And Receptions Over A Video Sensor Network, The Video File Contents Need To Be Encoded Packet By Packet Before Its Transmission Over Network. The Quality At The Receiver Side Can Be Maximized By Designing The Cross Layer System. The Transmission Rate And Encoding Rate Can Be Controlled By The Cross Layer System. The Fairness Of The Received Video Quality Can Be Developed By The Rate Controller. Instead, An Adaptive Parity Scheme That Drops Samples In Error Is Proposed And Shown To Improve Video Quality. Finally A Low Complexity, Adaptive Video Encoder, Is Proposed That Performs Low Complexity Motion Estimation On Sensors And Implemented To Further Optimize Energy Consumption., Thus Greatly Reducing The Amount Of Data To Be Transmitted And Video File Can Be Decompressed.

Index Terms: Compression, Multimedia Streaming, Congestion Control, Sensor Networks, Rate Controller.
\end{abstract}

\section{Introduction}

Wireless Multimedia Sensor Networks[1] are Self-Organizing Wireless Systems Of Embedded devices deployed to Retrieving the Audio and Video Streams that are distributively Process in the Real-Time Environment ,Store, Correlate and Fuse the Multimedia Applications that are Originating From Heterogeneous Sources. In Recent Years ,the Demand for Video Streaming Compression Applications [2][3] has been rise due to the Significant Increase in both the Bandwidth of the Wireless Channels And the Computational Power of Mobile Devices. A Higher Packet Loss Ratio and Variation of the Wireless Channels, along with the Heterogeneity of the users, make Video Streaming Compression over Wireless Sensor Networks a Challenging Problem. Wireless Multimedia Sensor Networks will enable new Applications including Multimedia Surveillances, Storage And Subsequent retrieval Of Potentially relevant activities. Video Compression is nothing But reducing the Large Sized Video File in to small sized Video file. Compression is very important due to the large amount of the Multimedia data especially in the Video. Video Compression Technologies are used to reducing and removing the Redundant Information so that the file can be effectively sent over a Network. Video Compression basically means Reducing the Video data mentioned previously a Digitized Video Sequence can compress up to $165 \mathrm{mps}$ of Data. There are two basic categories of compression. Lossless Compression is a class that will allow for the exact Original data to be Reconstructed From the Compressed Data. That means a Limited amount of Techniques are made available for Reducing the data so that result is Limited for Reduction of the data.GIF is an Example of Lossless Video Compression, but because of its limited abilities that are not relevant in the Video Compression Surveillance. In this paper, we study the potential of Compression Video Streaming for Wireless Multimedia Sensor Networks by conducting a Cross-Layer Performance Evaluation of Wireless Streaming of the Compressed Sensing Video on the Resource constrained Devices. Our objective is to design a Cross-Layer System that Jointly Controls the Video Encoding Rate. The Transmission of Video Streaming in Wireless Networks and their Statistical Traffic Characterization are Substantially Unexplored. For this reason a New Distributed Cross Layer Control Algorithm Compressive Distortion Minimizing Rate Control introduced That Jointly Regulates the Sampling rate, the Data rate that injected in the Network and the Rate of a Parity based Channel Encoder in order to maximize the Received Video Quality over a multi-Hop Wireless 
Network with Lossy Links. In the Cross-Layer Architecture the Compressive Video coding can be controlled at Application Layer, the rate at Transport Layer and Adaptive Parity at the Physical Layer.

\section{Related Work}

The Potential Compressed Sensing [3] has been investigates for Video Streaming in Wireless Multimedia Sensor Networks. The Rate Control[4] scheme is designed in order to Maximize the received video quality and to prevent the Network congestion while maintaining fairness between multiple video transmissions at the Receiver Side. Video Distortion is represented through Analytical and Empirical Models and at the Physical Layer based on the estimated channel quality the video Encoding rate and channel coding rate can be estimated. In order to avoid Congestion while maintaining fairness in the Domain of video quality rather than data rate the End-To-End Data rate is regulated [7],[8],[9].

In paper [5] [6] a new rate allocation algorithm is designed for Transform Domain Wyner-Ziv Video Coding (WZVC) without Feedback. Its objective is to design Intra-Frame Encoding and Complex Inter-Frame Decoding that is based on the Slepian-Wolf And Wyner-Ziv Distributed Source Coding Theorems. To allocate oroper Number of bits to Each Frame, Most Existing Wyner-Ziv Video Coding Solutions need a Feedback Channel (FC) at the Decoder. However, in many Video Coding Applications, the FC is not allowed. Moreover, the FC will introduce Latency and also the increase of Decoder complexity because several iterative decoding operations may be needed to decode the data to achieve target Video Quality. The Algorithm predicts the Number of bits for each Wyner-Ziv frame at the encoder as a Function of the Coding mode and the Quantization Parameters. Such Predictions will not significantly increase the complexity at the encoder. However, the prediction will be able to properly select the best mode and Quantization Parameter For encoding each Wyner-Ziv frame. Experimental Results show that the Algorithms is able to achieve good encoder rate allocation while still maintains consistent coding efficiency. Comparing To The WZVC[7] Coder With FC, this New WZVC Coder Without FC Induces only a small loss in Rate-Distortion Performance.

\section{Proposed System}

The Following Proposed System Consists of Problem Definition, Software Architecture and finally the proposed Solution.

\subsection{ProblemDefinition:}

In existing layered protocol stacks based on the IEEE 802.11 and 802.15.4 standards, frames are split into multiple packets. If even a single bit is attacked due to some of the channel errors, after a cyclic redundancy check, so that the entire packet will be dropped at a final or intermediate node. This can cause the video decoder is unable to decode an independently coded (I) frame, this leads to loss of the entire sequence of video frames.

\subsection{Software Architecture:}

The figure shows that the architecture of a Video Streaming Compression for Wireless Multimedia Sensor Networks. At The Application Layer, the Video file from the source is Compressed and divided in to ' $\mathrm{N}$ ' number of packets and then each packet is encoded, at Transport Layer the Congestion-Distortion Optimized Scheduling takes fully advantage of this Rate Allocation to select and transmit the packets of the Video stream which will maximize video quality. Next the Network Layer is responsible for packet Forwarding including routing _. Through An intermediate routers, then the Data Link Layer is Responsible for Media Access Control, Flow Control of the Video and then Error Checking .At the destination, the information is decoded and decompressed to obtain the original video file. 
The software architecture of the proposed system is shown below:

Source node

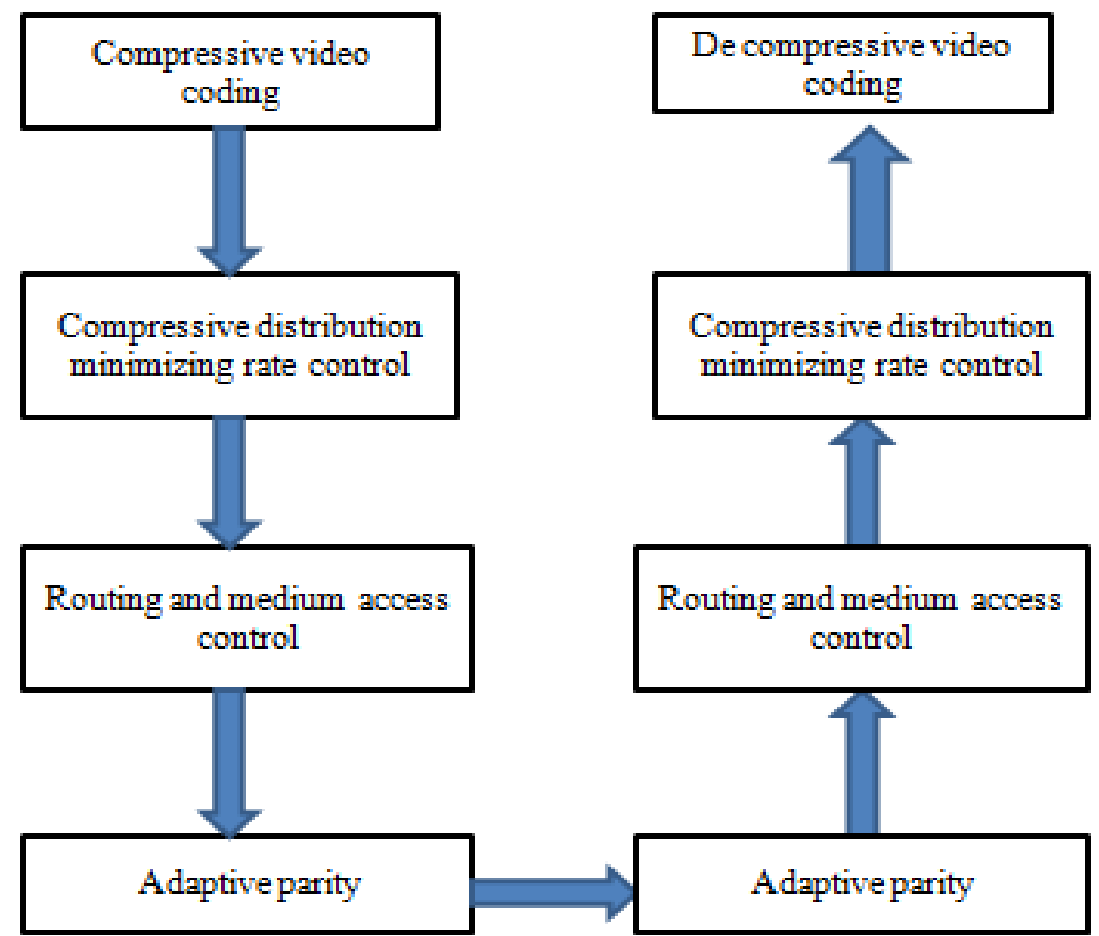

Fig 1 : An Architecture Of A Video Streaming Compression For Wireless Multimedia Sensor Networks.

\subsection{Proposed solution:}

\subsubsection{Compressed Video Transmission:}

Sender selects the Compressed File that needs to be send to the receiver based on the Temporal Correlation at Low complexity. Here the Sender Node browse the Video File that already in the Compressed Format in-Order to reduce the size of the file so that file can be Sent to the Network effectively.

\subsubsection{Video-On-Demand:}

The Compressed Video File is splitting in to 'N' Number of packets based on the Video-OnDemand. The packets are forwarded to the Buffer. The Packets Perform the action based on FIFO Queue. The First Packet added to the Queue is the first packet to be removed. So that the Video File can be received in FIFO order at the Receiver Side. The FIFO Receives Raw Video Data, buffers the raw capture Video Data at a rate, and transfers the raw capture Video data to the High Speed Memory. The Host FIFO Receives display Video Data from a remote source, Buffers the Display Video Data, and Transfers the input display Video data to the High Speed Memory. The PB FIFO reads capture Video Data and Display Video Data, During The PB Service, from the High Speed Memory to Provide an Input Queue for Video Compression and Video Decompression. The Display FIFO Receives, during the Display Service, Display Video Data from The High Speed Memory, and Buffers the Display Video data to Provide the Display Video Data at a Display Rate for the Host. 


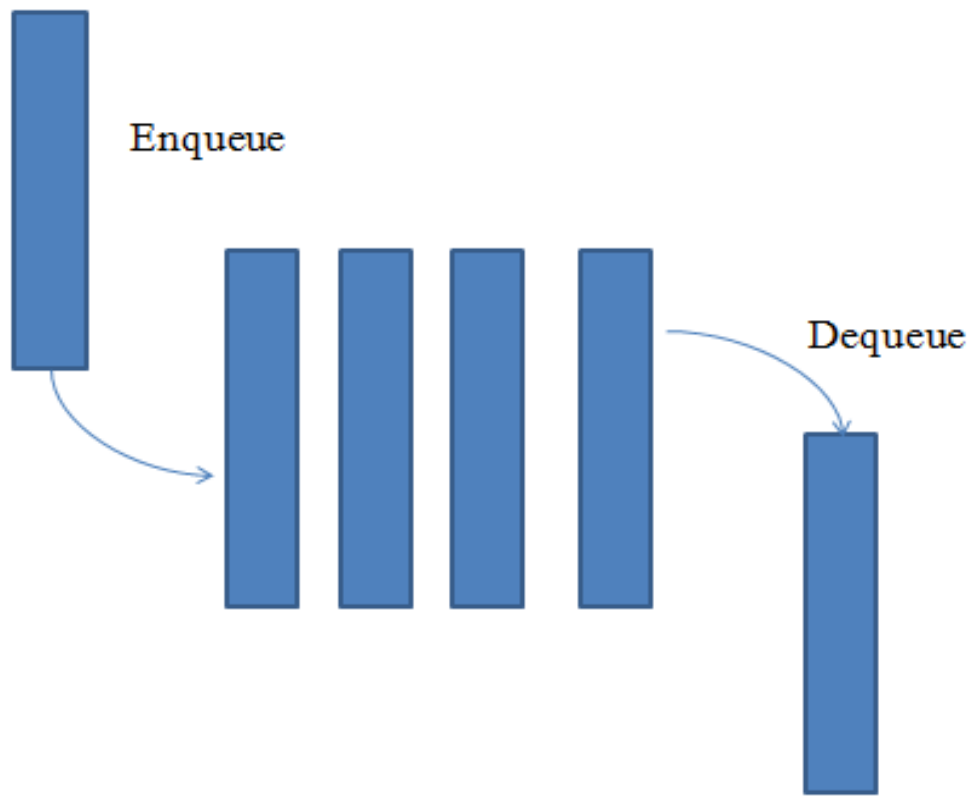

Fig 2:Fifo Queue

\subsubsection{Video Encoder:}

Key Establishment in Sensor Networks is a Challenging Problem because Asymmetric Key Cryptosystems are Unsuitable for use in Resource Constrained Sensor Nodes and also the Nodes could be physically compromised by an Attacker. For Each and Every Splitted Packet an Unique ID can be Assigned. So that the Video File can be Send Effectively to the Receiver. There are Three types of General Key Agreement Schemes.1.Trusted-Serverscheme.2.Self-Enfourcing Scheme.3.Key Pre-Distribution Scheme. In this paper Key Pre-Distribution Scheme is Used. In Key Pre-Distribution Scheme the Information of the Secret Key is Distributed among all the Sensor Nodes prior to deployment. If we know the nodes that are in the same Neighborhood before Deployment, the Keys can be decided a priori.In this Paper we are concentrating on RSA Cryptographic Technique. Here in this Technique Key Generation is one of the important part, where we need to Generate both Public Key and Private Key. The Sender will be Encrypting the Packet with Receiver's Public Key And Receiver will be Decrypting using his own Private Key.

RSA Algorithm: RSA is an Algorithm Used Or Public Key Cryptography.

Step 1: Select Random Prime Numbers $P$ And $Q$, And Check That $\mathrm{P} !=\mathrm{Q}$

Step 2 :Compute Modulus $N=\mathrm{Pq}_{-}$

Step 3: Compute $\Phi(\mathrm{N})=\Phi(\mathrm{P}) \Phi(\mathrm{Q})=(\mathrm{P}-1)(\mathrm{Q}-1)$

Step 4: Select Public Exponent E, Such That $1<\mathrm{E}<\Phi(\mathrm{N})$ And $\operatorname{Gcd}(\mathrm{E}, \Phi(\mathrm{N}))=1$

Step 5:Compute Private Exponent D = E-1 $(\operatorname{Mod} \Phi(\mathrm{N}))$

Step 6: Public Key Is $\{N, E\}$, Private Key Is $D$

Encryption: $C=\operatorname{Memod} N$,

Decryption: $M=C d \bmod N$

Digital Signature: $S=H(M) \operatorname{Dmod} N$, Verification: $M^{\prime}=\operatorname{Semod} N$, If $M^{\prime}=H(M)$ Signature Is Correct. H Is A Publicly Known Hash Function.

\subsubsection{Active Buffer Management:}

In this paper Active Buffer Management Scheme is used the Transmission of Video over IP based Networks requires the Segmentation of Video Data into IP Packets. The Maximum Size of an IP Packet as a Constant Parameter of the Networks along the routing path. Generally it is smaller than the average size of a 
video frame. Therefore video frames is segmented into several IP packets during Transmission Process. Here the Active Buffer Management is used to filter the packets and it En-Route the packets to the Destination. There are Two types of Buffer Management 1.Packet Based Strategies where each incoming Packets is Treated Individually.2.Frame Based Strategies where each Video Frame is an Atomic Unit during the Buffer Management Decision and Actions.

\subsubsection{Adaptive Parity Check:}

The Adaptive Parity Check is used to measure or estimate the sample Error Rate of the channel to determine a parity Scheme for encoding the samples, which are the input directly from the Video encoder. The Adaptive Parity Check, checks whether the Mac for each packet at the receiver side. If the mac matches then Receiver receives Packet Successfully. If not that Packet is dropped so the file cannot be decoded Successfully.

\section{Results}

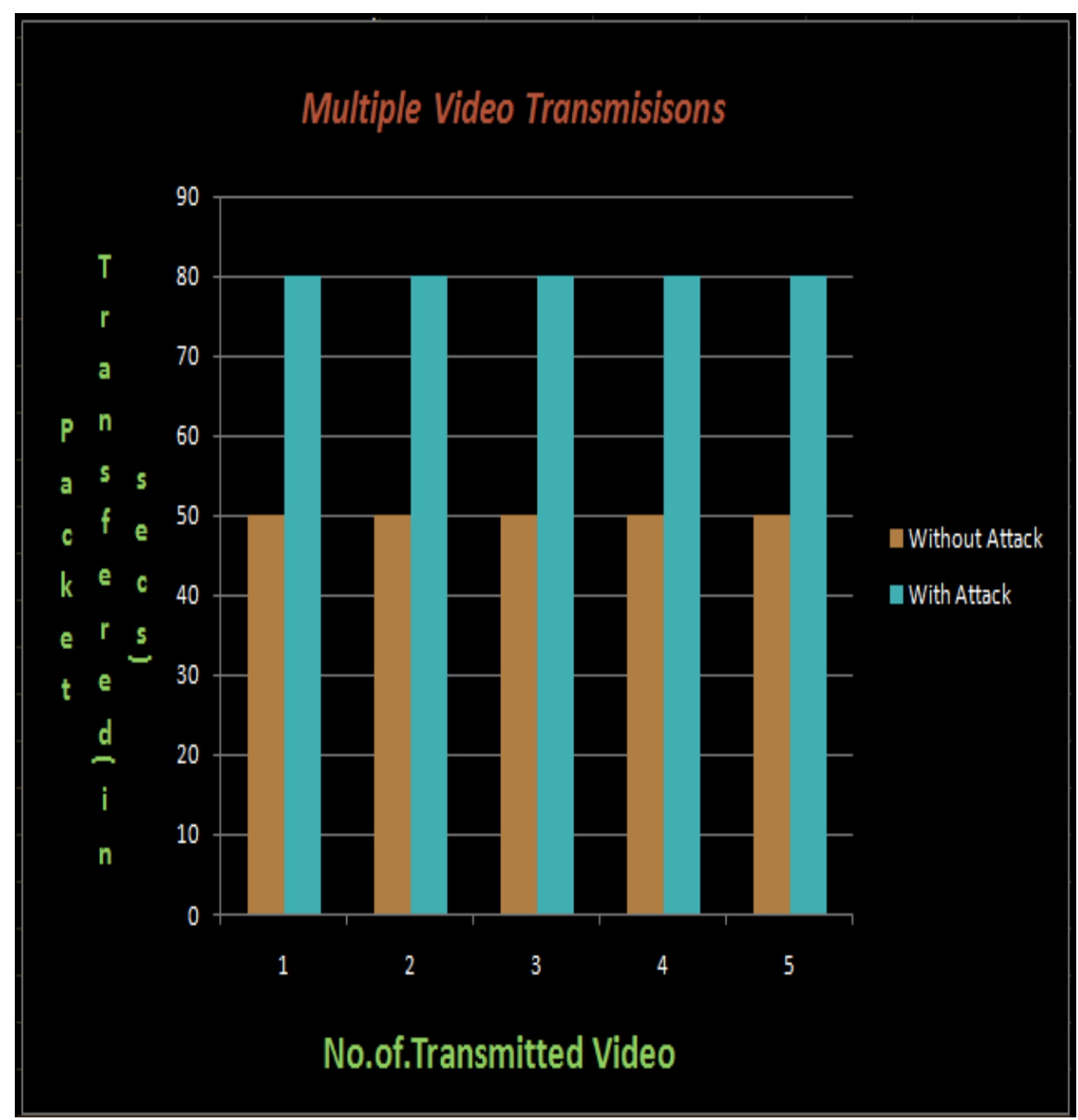

Fig 4.1: Video Streaming Compression Results.

\section{Conclusion}

In this paper we addressed the problem of packets dropped at an intermediate node or final node. Where the attacker is a part of the Network who is aware of Network secrets and also the implementation details. In order to Overcome the problem of dropped Packets we develop Adaptive Rate Control Scheme that Reconfigure the dropped Packets. We Analyze the Security of our schemes and through simulation we can achieve the higher throughput by Re-sequencing the dropped Packets. 


\section{References}

[1] S. Pudlewski, T. Melodia, And A. Prasanna, "C-DMRC: Compressive Distortion-Minimizing Rate Control For Wireless Multimedia Sensor Networks," In Proc. Of IEEE International Conference On Sensor, Mesh And Ad Hoc Communications And Networks (SECON) 2010, Boston, MA, June 2010.

[2] I. F. Akyildiz, T. Melodia, And K. R. Chowdhury, “A Survey On Wireless Multimedia Sensor Networks,” Computer Networks (Elsevier), Vol. 51, No. 4, Pp. 921-960, Mar. 2007.

[3] A. Aaron, S. Rane, R. Zhang, And B. Girod, “Wyner-Ziv Coding For Video: Applications To Compression And Error Resilience," In Proc. Of IEEE Data Compression Conf. (DCC), Snowbird, UT, March 2003, Pp. 93-102.

[4] D. Donoho, “Compressed Sensing,” IEEE Transactions On Information Theory, Vol. 52, No. 4, Pp. 1289-1306, Apr. 2006.

[5] B. Girod, A. Aaron, S. Rane, And D. Rebollo-Monedero, “Distributed Video Coding," Proc. Of The IEEE, Vol. 93, No. 1, Pp. 71-83, January 2005.

[6] T. Sheng, G. Hua, H. Guo, J. Zhou, And C. W. Chen, “Rate Allocation For Transform Domain Wyner-Ziv Video Coding Without Feedback," In ACM Intl. Conf. On Multimedia, New York, NY, USA, October 2008, Pp. 701-704.

[7] J. Romberg, 'Imaging Via Compressive Sampling, ” IEEE Signal Processing Mag Azine,Vol.25,No.2,Pp.14-20,2008

[8] M. Duarte, M. Davenport, D. Takhar, J. Laska, T. Sun, K. Kelly, And R. Baraniuk, "Single-Pixel Imaging Via Compressive Sampling," IEEE Signal Processing Magazine, Vol. 25, No. 2, Pp. 83-91, 2008.

[9] M . Wakin, J. Laska, M. Duarte, D. Baron, S. Sarvotham, D. Takhar, K. Kelly, And R. Baraniuk, “Compressive Imaging For Video Representation And Coding," In Proc. Picture Coding IEEE COMSOC MMTC E-Letter 26/30 Vol.4, No.9, October 2009 Symposium (PCS), April 2006. 